\title{
Asteroseismic search for invisible binary companions
}

\author{
Hiromoto Shibahashi ${ }^{1}$, Simon J. Murphy ${ }^{2,3}$ and Donald W. Kurtz ${ }^{4}$ \\ ${ }^{1}$ Department of Astronomy, University of Tokyo, \\ Bunkyo-ku, Tokyo 113-0033, Japan \\ email: shibahashi@astron.s.u-tokyo.ac.jp \\ ${ }^{2}$ Sydney Institute for Astronomy, School of Physics, University of Sydney, \\ NSW 2006, Australia \\ ${ }^{3}$ Stellar Astrophysics Centre, Department of Physics and Astronomy, Aarhus University, \\ 8000 Aarhus C, Denmark \\ email: murphy@physics.usyd.edu .au \\ ${ }^{4}$ Jeremiah Horrocks Institute, University of Central Lancashire, \\ Preston PR1 2HE, UK \\ email: dwkurtz@uclan.ac.uk
}

\begin{abstract}
Continuous and precise space-based photometry has made it possible to measure the orbital frequency modulation of pulsating stars in binary systems with extremely high precision over long time spans. We present the phase modulation (PM) method for finding binaries among pulsating stars. We demonstrate how the orbital elements of a pulsating binary star can be obtained analytically from photometry alone, without spectroscopic radial velocity measurement. Frequency modulation (FM) caused by binary orbital motion also manifests itself in the Fourier transform, as a multiplet with equal spacing of the orbital frequency. The orbital parameters can also be extracted by analysing the amplitudes and phases of the peaks in these multiplets. We derive analytically the theoretical relations between the multiplet properties and the orbital parameters, and present a method for determining these parameters, including the eccentricity and the argument of periapsis. This, too, is achievable with the photometry alone, without spectroscopic radial velocity measurements. We apply these two methods to Kepler mission data and demonstrate that the results are in good agreement with each other. These methods are used to search for invisible binary companions, including planets and invisible massive objects such as neutron stars and stellar-mass black holes.
\end{abstract}

Keywords. asteroseismology, stars: oscillations, techniques: miscellaneous

\section{Introduction}

Binary orbital motion causes a periodic variation in the path length travelled by light emitted from a star and arriving at Earth. Hence, if the star is pulsating, the observed phase of the pulsation varies over the orbit (see Sect.2). Conversely, once we have observed such phase variation, or multiplets in the frequency spectrum (see Sect.4), we can extract information about the binary orbit from photometry alone. This problem was first discussed by Woltjer (1922) and later by Irwin (1952). However, the required photometric and timing precision was not reachable at that time, hence application of their methods was not practical. In contrast, recent space-based photometry has made it possible to measure the orbital phase variation of pulsating stars in binary systems with extremely high precision over long time spans. This variation also manifests itself in the Fourier transform, as a multiplet with spacing equal to the orbital frequency. We have developed two complementary methods for this problem: one, called the FM method, dealing with 
modulation in the frequency domain (Shibahashi \& Kurtz 2012†, Shibahashi, Kurtz \& Murphy 2015; see also Koen 2014), and the other, called the PM method, analyzing the phase modulation in the time domain (Murphy et al. 2014, Murphy \& Shibahashi 2015; see also Balona 2014). In this paper, we outline how the orbital elements of a pulsating binary star can be obtained analytically. These methods are used to search for invisible binary companions in Kepler data, including planets and invisible massive objects such as neutron stars and stellar-mass black holes.

\section{The light travel time effect}

Let us consider a star sinusoidally pulsating with a single angular frequency $\omega_{0}$ in a binary. The observed luminosity variation, whose amplitude is assumed to be unity, at time $t$ is then given by

$$
\Delta L(t)=\cos \left\{\omega_{0}\left[t-\frac{1}{c} \int_{0}^{t} v_{\text {rad }}\left(t^{\prime}\right) \mathrm{d} t^{\prime}\right]\right\}
$$

where $c$ is the speed of the light and $v_{\text {rad }}(t)$ denotes the radial velocity, due to the orbital motion, of the pulsating star at the time $t$, where the epoch is the time at which the star passes the nodal point directed towards us. The second term in the square bracket on the right-hand side, which is hereafter denoted as $\tau$, is the the time delay caused by the light travel time effect. With knowledge of celestial mechanics, $\tau$ is expressed as a series of sine functions with the orbital angular frequency $\Omega$ (see Shibahashi, Kurtz \& Murphy (2015) for more details):

$$
\Delta L(t)=\cos \left[\left(\omega_{0} t+\phi\right)+\alpha \sum_{n=1}^{\infty} \xi_{n} \sin \left(n \Omega t+\vartheta_{n}\right)\right],
$$

where $\alpha:=a_{1} \omega_{0} \sin i / c$ is the ratio between the light travel time across the projected semi-major axis, $a_{1} \sin i / c$, and the pulsation period of the mode in consideration, $\xi_{n}$ and $\vartheta_{n}$ are functions of the eccentricity, $e$, the angle between the nodal point and the periapsis, $\varpi$, and the time of periapsis passage, $t_{\mathrm{p}}$.

The larger the orbit, the larger the light travel time effect. Thus, the combination of high pulsation frequency and long orbital period is favourable to large values of $\alpha$.

\section{PM method}

In the PM method, first of all, the pulsation frequencies are measured in the Fourier transform. Then the light curve is divided into short segments and, with the frequencies fixed, the pulsation phase in each segment is measured by a least-squares method. This provides us with the time delays (TDs) as a function of time (see the upper panel of Fig. 1). The orbital angular frequency $\Omega$ is obtained from the Fourier transform of the time delays (the lower panel of Fig. 1). The presence of harmonics indicates that the orbit deviates from a circle. The amplitude ratio between the first two components provides us with a good initial guess for $e$. The epochs at which the TD curve reaches its maxima and minima correspond to the orbital phase at which the star passes the farthest point and the nearest point from us, respectively. Hence the eccentric anomalies of these two points are different from each other by $\pi$ radians. This constraint allows us to determine the time of periapsis passage, $t_{\mathrm{p}}$. Once the orbital phase of periapsis passage is determined, the true anomalies at the nearest point and at the farthest point, at which $v_{\text {rad }}$ should be zero, are

$\dagger$ Their table 4 is corrected in Shibahashi \& Kurtz (2013). 

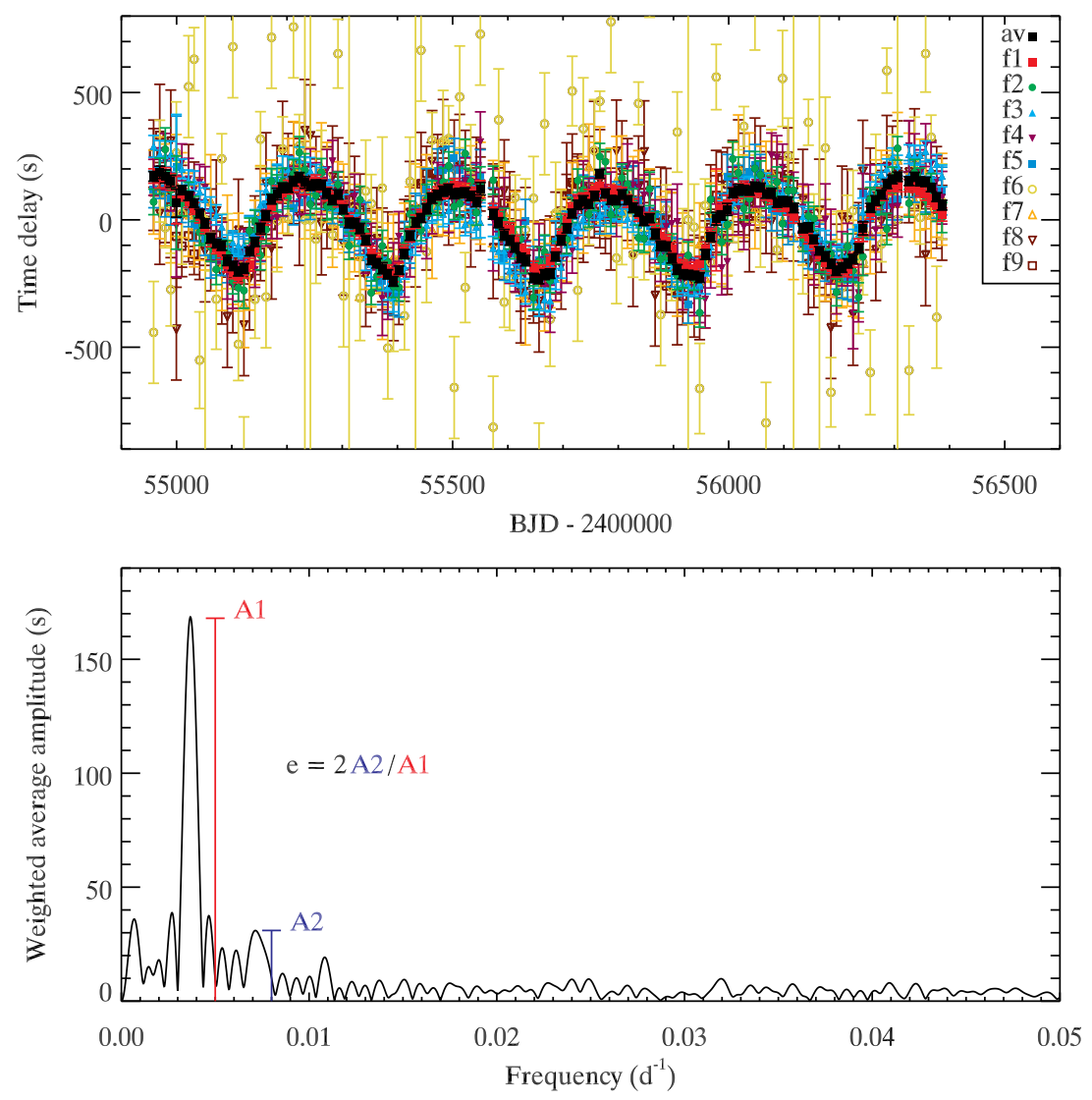

Figure 1. Upper panel: An example of a TD curve (KIC 9651065) using nine different pulsation modes, including one in the super-Nyquist frequency range (cf. Murphy, Shibahashi \& Kurtz 2013). The weighted average is shown as filled black squares. Lower panel: Fourier transform of the TD curve shown in the left panel. The peak frequency provides us the orbital frequency, and the amplitude ratio between the two highest peaks gives the eccentricity. Both panels were reproduced from Murphy \& Shibahashi (2015).

then determined. Whether $v_{\mathrm{rad}}=0$ is fulfilled for the given values of true anomalies and eccentricity is dependent on the value of periapsis $\varpi$. Then, the requirement of $v_{\text {rad }}=0$ at the extrema of the TD curve gives the value of $\varpi$. Once $e$ and $\varpi$ are determined, the projected semi-major axis, $a_{1} \sin i / c$, is determined with the help of the difference between the extrema in $\tau, \tau_{\max }-\tau_{\min }$, by $a_{1} \sin i / c=\left(\tau_{\max }-\tau_{\min }\right)\left(1-e^{2} \cos 2 \varpi\right)^{-1 / 2} / 2$. For more details with more graphical display, see Murphy \& Shibahashi (2015).

With the initial guesses for the orbital parameters thus obtained, we get a reasonable TD curve that fits the observations. We may search for the best-fitting values of the orbital parameters by minimizing the sum of the squares of the residuals, $\chi^{2}$-fitting, or by Markov chain Monte Carlo methods (the left panel of Fig. 2). The mass function is eventually derived from $\Omega$ and $a_{1} \sin i / c$. The radial velocity (RV) is obtained by $v_{\text {rad }}=c \mathrm{~d} \tau / \mathrm{d} t$, but it is provided only as a visualization (the right panel of Fig. 2): the computation of radial velocities is not a necessary step in solving the orbit. 

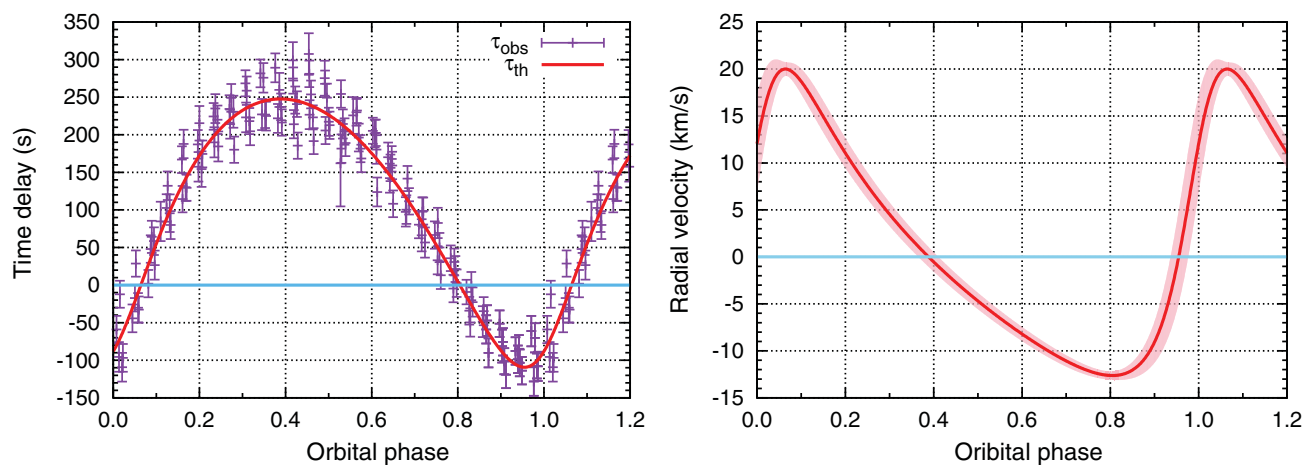

Figure 2. Left panel: The best-fitting TD curve for KIC 9651065. The periapsis passage was chosen as the orbital phase of zero. Reproduced from Murphy \& Shibahashi (2015). Right panel: The photometrically obtained RV of KIC 9651065 . This is provided only as a visualization, and is not required for the derivation of the orbital parameters.
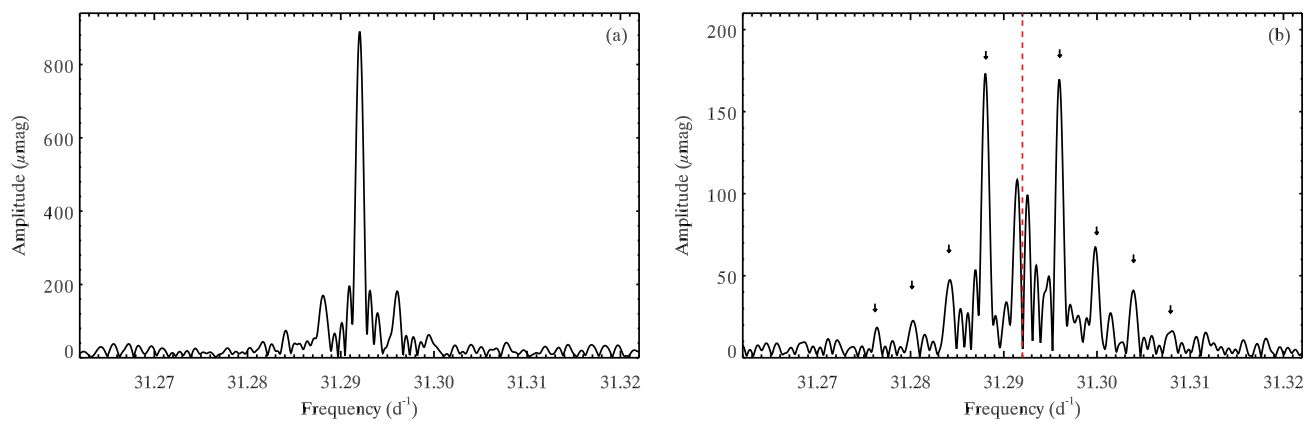

Figure 3. (a) The amplitude spectrum for KIC 8264492, around the highest peak. Sidelobes are visible without pre-whitening the central peak, but in panel (b) the same region is shown with the central peak pre-whitened (at the dashed red line). Arrows show four pairs of sidelobes. Reproduced from Shibahashi, Kurtz \& Murphy (2015).

\section{FM method}

The Fourier transform of the luminosity variation given in equation (2.2) leads a frequency multiplet around the intrinsic frequency $\omega_{0}$ in the frequency spectrum, and each component of the multiplet is separated from its neighbouring components by the orbital frequency $\Omega$ (see Fig. 3):

$$
\Delta L(t)=\Re\left\{\mathcal{A}_{0} \mathrm{e}^{\mathrm{i} \omega_{0} t}+\sum_{m=1}^{\infty}\left(\mathcal{A}_{+m} \mathrm{e}^{\mathrm{i}\left(\omega_{0}+m \Omega\right) t}+\mathcal{A}_{-m} \mathrm{e}^{\mathrm{i}\left(\omega_{0}-m \Omega\right) t}\right)\right\},
$$

where $\mathcal{A}_{0}$ and $\mathcal{A}_{ \pm m}$ are the complex amplitudes. For $\alpha<1$, the eccentricity $e$ is estimated from the amplitude ratio between the second sidelobes $\left(A_{ \pm 2}\right)$ and the first sidelobes $\left(A_{ \pm 1}\right)$. On the other hand, the ratio between the first sidelobes and the central component gives $\alpha \xi_{1}$. The phase difference between the average of the first sidelobes, $\left(\phi_{+1}+\phi_{-1}\right) / 2$, and the central component, $\phi_{0}$, is dependent on the angle between the nodal point and the periapsis, $\varpi$, and $\alpha \xi_{2}$. The same is true for the relative difference of the amplitude of the first sidelobes, $\left(A_{+1}-A_{-1}\right) /\left(A_{+1}+A_{-1}\right)$, and their $\alpha \xi_{2}$-dependence is in common. So, the value of $\varpi$ can be extracted from the first sidelobes and the central component. Once the values of $e$ and $\varpi$ are obtained, we estimate $\xi_{1}(e, \varpi)$. The value of $\alpha$ is obtained by dividing $\alpha \xi_{1}$ by $\xi_{1}$, and then the projected semi-major axis is derived by 

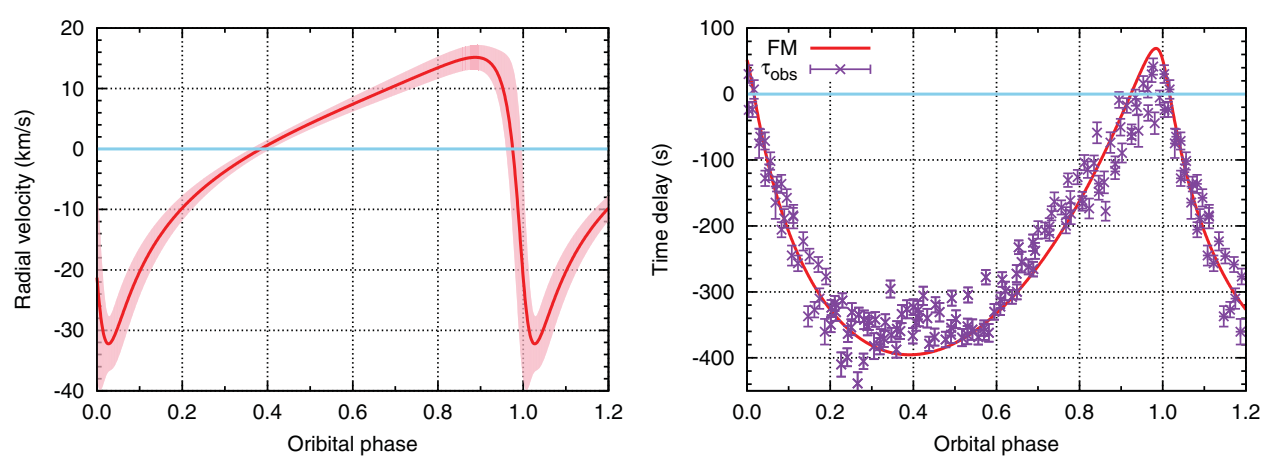

Figure 4. Left panel: The RV curve, derived from the Kepler photometric data with the FM method, of KIC 8264492 . The periapsis passage was chosen as the orbital phase of zero. Right panel: The TD curve of KIC 8264492, derived with the FM method. It is in good agreement with TDs derived by the PM method. Taken from Shibahashi, Kurtz \& Murphy (2015).

$a_{1} \sin i / c=\alpha / \omega_{0}$. We may iterate the above-mentioned process. The mass function is eventually derived from the parameters thus obtained. For more details with more graphical display, see Shibahashi, Kurtz \& Murphy (2015).

The RV curve is derived from the orbital parameters thus obtained (the left panel of Fig. 4). The TD is also derived with the FM method. The right panel of Fig. 4 shows that the TD thus obtained is in good agreement with the observed TDs.

We have also demonstrated the validity of the FM method by showing the consistent results obtained from it when compared to a traditional eclipsing binary light curve analysis (Kurtz et al. 2015).

\section{Discussion}

Historically, detection of an invisible companion was first successfully done by astrometry (Bessel 1844), and then by spectroscopy (Vogel 1890, Pickering 1890). Spectroscopic observations of the Doppler shift of spectral lines have been widely used for exoplanet hunting. A promising application of our photometric methods is in the search for exoplanets or brown dwarfs orbiting pulsating upper main-sequence stars. It is expected, from our simulation, that companion stars in the mid-to-low mass range of brown dwarfs are detectable in the case of $2 M_{\odot} \delta$ Sct stars. Another application is at the opposite extreme, in the search for invisible massive companions in binary systems. We know of binary systems composed of a neutron star or a stellar-mass black hole and either an early-type massive star, or a cool star. Hence it seems natural to expect a binary system of a neutron star or a stellar-mass black hole and an A-type $\delta$ Sct star. The present methods provide a unique, promising way of finding such exotic systems.

\section{References}

Balona, L. A. 2014, MNRAS, 443, 1946

Bessel, F. W. 1844, MNRAS, 6, 136

Irwin, J. B. 1952, ApJ, 116, 211

Koen, C 2014, MNRAS, 444, 1486

Kurtz, D. W., Hambleton, K. M., Shibahashi, H., Murphy, S. J., \& Prša, A. 2015, MNRAS, 446, 1223

Murphy, S. J., Bedding, T. R., Shibahashi, H., Kurtz, D. W., \& Kjeldsen, H. 2014, MNRAS, 441, 2515 
Murphy, S. J. \& Shibahashi, H. 2015, MNRAS, 450, 4475

Murphy, S. J., Shibahashi, H., \& Kurtz, D. W. 2013, MNRAS, 430, 2986

Pickering, E. C. 1890, MNRAS, 50, 269

Shibahashi, H. \& Kurtz, D. W. 2012, MNRAS, 422, 738

Shibahashi, H. \& Kurtz, D. W. 2013, ASP-CS, 479, 503

Shibahashi, H., Kurtz, D. W., \& Murphy, S. J. 2015, MNRAS, 450, 3999

Vogel, H. C. $1890, A N, 123,289$

Woltjer, J., Jr. 1922, BAN, 429, 3472 